3. LARGE-SCALE STRUCTURE AND KINEMATICS 


\title{
AN OVERVIEW OF THE STRUCTURE AND KINEMATICS OF THE MAGELLANIC CLOUDS
}

\author{
B.E. WESTERLUND \\ Astronomical Observatory \\ Box 515, S-751 20 Uppsala \\ Sweden
}

\begin{abstract}
A vast amount of observational data concerning the structure and kinematics of the Magellanic Clouds is now available. Many basic quantities (e.g. distances and geometry) are, however, not yet sufficiently well determined. Interactions between the Small Magellanic Cloud (SMC), the Large Magellanic Cloud (LMC) and our Galaxy have dominated the evolution of the Clouds, causing bursts of star formation which, together with stochastic self-propagating star formation, produced the observed structures. In the youngest generation in the LMC it is seen as an intricate pattern imitating a fragmented spiral structure. In the SMC much of the fragmentation is along the line of sight complicating the reconstruction of its history. The violent events in the past are also recognizable in complex velocity patterns which make the analysis of the kinematics of the Clouds difficult.
\end{abstract}

\section{Introduction}

To understand the observed structures and kinematics of the Magellanic Clouds (MCs) it is necessary to know something of their history. For example, is the occurrence of the LMC and the SMC in the neighbourhood of our Galaxy a chance meeting with the Clouds just passing by (cf. Mathewson \& Ford 1984) or have the Clouds formed as part of our Hypergalaxy (cf. Einasto et al. 1976)? Neither hypothesis appears likely. More probably, they formed in our Local Group of Galaxies and were caught by our Galaxy during the expansion of the Group. Some of the other small members of the Group escaped the gravitational forces of M31 and the Galaxy (cf. Mishra 1985). They are all of type Im or S. Most of the others, all now of type dE, were caught early by either of the two giants and stripped of their gas and dust. The MCs entered our Hypergalaxy later and have managed to keep most of the gas. Normal galactic nuclei and bulges probably began to form but tidal forces distorted them into the bars we see today. These massive bars have caused gas clouds to fall towards them with star burst conditions as a result (Larson 1987).

The LMC and the SMC form a Magellanic System which moves in the plane of the Local Group. The most obvious results of the interaction between the Galaxy, the LMC and the SMC are the Magellanic Stream, the Inter-Cloud (IC) region gas and, maybe, the split of the SMC into two nearly equal parts (SMCRemnant and MiniMC (Mathewson et al. 1987)). Effects are also seen in stellar generations in the Clouds and in our Galaxy (Scalo 1987).

From the current positions and velocities of the Clouds, Murai \& Fujimoto (1980, see also Fujimoto \& Murai 1984) have determined their motions $10 \mathrm{Gyr}$ back in time. Particularly important are the epochs when the Clouds had close encounters and when they came close to the Galaxy. Such events occurred 7.5, 2.6, 1.5 and $0.2 \mathrm{Gyr}$ ago with the SMC coming within 2 - 7 $\mathrm{kpc}$ from the LMC, and $0.04 \mathrm{Gyr}$ ago when the Clouds were in their perigalacticon. At these epochs strong tidal forces caused violent bursts of star formation at several locations in the Clouds, 
producing the dominating generations (see Westerlund 1990). The bursts were followed by stochastic self-propagating star formation (SSPSF) which lasted as long as conditions were suitable.

\section{The distances of the Magellanic Clouds}

The position of an object in the Clouds was previously ignored in distance determinations but is now considered important. Many new determinations of the distances of the MCs exist (see Westerlund 1990). The most likely distance modulus of the LMC appears to be $(\mathrm{m}-\mathrm{M})_{0}=18.5 \pm$ 0.15 mag. The SMC is 0.31 mag more distant than the LMC when Cepheids and RR Lyr stars are considered but $0.6-0.8$ mag when early-type stars are used. This discrepancy may be partly caused by the pronounced extension of the SMC in depth; $(\mathrm{m}-\mathrm{M})_{\mathrm{o}}=18.9 \mathrm{mag}$ may be a reasonable mean value for the SMC. The linear distance of the LMC is then $50 \mathrm{kpc}$, and of the SMC $60 \mathrm{kpc}$. The SMC may have a depth of $\pm 10 \mathrm{kpc}$ and also the LMC may be appreciably extended along the line of sight (Songaila et al. 1986)

\section{The geometry of the Magellanic Clouds}

\subsection{THE INCLINATION OF THE LMC}

The LMC has long been regarded as a thin flat disk seen nearly face-on (de Vaucouleurs \& Freeman 1973). The tilt of the LMC plane to the plane of the sky was determined to $i=27^{\circ} \pm 2^{\circ}$ on the basis of optical and $21 \mathrm{~cm}$ isophotes and the surface distribution of clusters, assuming that the untilted distributions are circular. This assumption is doubtful in the case of an irregular galaxy with most population centroids as well as the proposed centre of rotation offset from the optical centre. It may apply to the older populations with centroids close to the optical centre but hardly to the Extreme Population I. Support for $i \approx 27^{\circ}$ has, however, been found in the distribution of some cepheids (see de Vaucouleurs 1980) but the errors in the mean moduli are large. Other investigations giving values close to $27^{\circ}$ have used populations truncated on the southern side of the LMC (e.g. Feitzinger et al. 1977) or accepted poorly defined solutions only because they fell in the range $30^{\circ} \leq i \leq 37^{\circ}$ (Prévot et al. 1989).

Determinations of the tilt, $i$, and the position angle of the line of nodes, $\theta$, have been summarized by Westerlund (1990, Table 3 ). The values on $i$ determined with the aid of extreme Population I are smaller than the others: this may be due to its very irregular distribution over the LMC. Recent determinations of $i$ with the aid of cepheids give values up to $45^{\circ}$. Visvanathan (1989) found that most of the dispersion in the P-L relation for LMC cepheids is due to the geometry: with $i=45^{\circ}$ the cepheids extend over a depth of $8 \mathrm{kpc}$. The true tilt of the main body of the LMC may thus be closer to $45^{\circ}$ than previously accepted. With this tilt and the eastern part of the LMC closest to us, the SMC falls in the LMC plane provided its $(\mathrm{m}-\mathrm{M})_{\mathrm{o}} \approx 19.1$, a value frequently used (cf. Laney \& Stobie 1986). 


\subsection{THE TRANSVERSE MOTION OF THE LMC AND ITS LINE OF NODES}

The position angle, $\theta$ of the line of nodes is relatively well determined. Feast et al. (1961) found $\theta_{k}=171^{\circ}$ from the maximum velocity gradient, a value that agreed with the geometrically determined value, $\theta_{g}$. Feitzinger et al. (1977) pointed out that a difference between $\theta_{g}$ and $\theta_{k}$ could be due to the transverse motion of the LMC. They derived a transverse velocity $V_{t}=275$ $\mathrm{km} \mathrm{s}^{-1}$ relative to the Sun using $\theta_{g}=168^{\circ}$ and $\theta_{k}=188^{\circ}$. The determinations of $\theta$ are heavily based on Population I objects. It is doubtful that the application of a weighting system can correct for the asymmetries in the central parts of the LMC. Lin and Lynden-Bell (1982) derived $V_{t}=373$ $\mathrm{km} \mathrm{s}^{-1}$ (for the mass centre of the LMC and the SMC) applying a different technique. Recently, Meatheringham et al. (1988) found $V_{t}=275 \mathrm{~km} \mathrm{~s}^{-1}$ from a study of planetary nebulae (PN), using the central heavily perturbed $2^{\circ}$ of the LMC to define the kinematical line of nodes. Prévot et al. (1989) found $V_{t}=150 \mathrm{~km} \mathrm{~s}^{-1}$ from their accurate radial velocities for red supergiants. The differences are due to different values of $\theta_{k}$. The direction of the transverse motion is in all cases parallel to the Magellanic Stream. Prévot et al. used a material well spread over the "Extreme Population I area" $\left(r \approx 3^{\circ}\right)$. They confirm Feitzinger's (1980) conclusion that $\theta$ is strongly variable. The variations are large enough to make them consider "as grounded (sic.) the revision of the morphology of the LMC with the help of suitable models".

\subsection{POPULATION CENTROIDS IN THE LMC}

Early $21 \mathrm{~cm}$ radio observations led to the adoption of a centre of rotation at $05^{\mathrm{h}} 2 \mathrm{O}^{\mathrm{m}},-68.8^{\circ}$ (Kerr \& de Vaucouleurs 1956), defined so that the velocity curve would be symmetrical for derivation of the LMC mass. The radio centre is displaced from the optical centre, at $05^{\mathrm{h}} 24^{\mathrm{m}},-69.8^{\circ}$; from the centroid of the projected $\mathrm{HI}$ distribution, at $05^{\mathrm{h}} 35^{\mathrm{m}},-68.5^{\circ}$; and from all other centroids. All the centroids of Extreme Population I objects lie to the north of those of the older ones, e.g. the PN, the novae and the outlying clusters. The near collision between the Clouds about $0.2 \mathrm{Gyr}$ ago pulled much of the gas in the LMC northeastwards. This is underlined by the fact that the centroid of the stellar Extreme Population I is at $05^{\mathrm{h}} 33^{\mathrm{m}}-67.7^{\circ}$ (de Vaucouleurs \& Freeman 1973, Table 2). This population is thus hardly suitable for defining the motions of the main body of the LMC.

\subsection{THE GEOMETRY OF THE SMC}

The early efforts to define the SMC as a rotating galaxy with a spiral structure (cf. de Vaucouleurs \& Freeman 1973) have been replaced by intense efforts to understand its extension in depth and its possible fragmentation. Determinations of $i$ and $\theta$ exist but may have little relevance if the extension of the SMC is mainly in depth with the Wing and the NE part closest to us. The same may apply to the centroids determined. There is a tendency for the older populations (novae, PN) to be displaced somewhat towards the West (see Westerlund 1990). 


\section{The kinematics of the Magellanic Clouds}

\subsection{THE KINEMATICS OF THE LMC}

The rotation curve derived from Extreme Population I objects, stars and HII regions, has been found to agree well with the (bumpy) HI rotation curve (Rohlfs et al. 1984). Young stars with deviating velocities do, however, exist. McGee (1964) identified 18 early-type supergiants with velocities and reddening indicating that they are outside the LMC plane, and Page and Carruthers (1981) noted several clusters and associations in front of or behind the HI. Prévot et al. (1989) were forced to exclude $6 \%$ of their stars in the determination of a rotation curve. Many attempts have been made to show that also older populations have rotation curves symmetrical around the radio centre. Meatheringham et al. (1988) derived a rotation solution for the PN in the LMC which, according to the authors, is essentially identical with that of the HI. Its centre of symmetry is half way between the optical and radio centres, and the vertical velocity dispersion is 19.1 $\mathrm{km} \mathrm{s}^{-1}$, much greater than the $5.4 \mathrm{~km} \mathrm{~s}^{-1}$ found for the HI. Regions with increased velocity dispersion in HI prove that star formation has stirred up the gas in the plane. These regions correspond to known supergiant shells. The velocity dispersion of the PN exceeds the values for the star-forming shells, possibly due to the initial velocities of the precursor stars. In starburst regions expansion velocities of $36 \mathrm{~km} \mathrm{~s}^{-1}$ have been observed (Dopita et al. 1985b). The PN may trace similar events of SSPSF in the past.

Freeman et al. (1983) have concluded that clusters younger than $1 \mathrm{Gyr}$ (most of them are younger than $0.1 \mathrm{Gyr}$ ) have motions similar to the gas in their vicinity and share the rotation solution found for the HI and HII. Also the intermediate-age clusters form a flattened system and it is possible that even the oldest clusters $(\geq 10 \mathrm{Gyr})$ do so. The material is, as pointed out by the authors, scanty.

Bessell et al. (1986) found that the intrinsic line-of-sight dispersion of the velocities of a group of long period variables (LPVs) in the LMC is $30 \mathrm{~km} \mathrm{~s}^{-1}$. They find this consistent with that expected for stars belonging to a flattened disk with a scale height of $\approx 0.3 \mathrm{kpc}$ and see also marginal evidence that the LPV system rotates. However, the fact that most of the stars are within $1^{\circ}$ of the $\mathrm{HI}$ centre of rotation where non-circular motions dominate, makes it difficult to accept this interpretation as the only one possible.

Hartwick \& Cowley (1988) have determined radial velocities for $39 \mathrm{CH}$ stars in the LMC. The resulting velocity distribution appears similar to that of the oldest clusters as well as to that of the LPVs and they may belong to the same age group. Whether they form a true halo system or a flattened disk system remains to be determined.

The general tendency in most studies of the kinematics of the populations of the LMC has been to establish the dominance of the HI centre of rotation. Instead of asking why the Bar is asymmetrically placed in the LMC we should ask why the Extreme Population I is so. An answer has been suggested above: the interaction between the two Clouds during the last close encounter pulled much of the gas northwards in the LMC.

\subsection{THE KINEMATICS OF THE SMC}

Radial velocities determined from the often multiple-peaked HI profiles in the SMC have been used to derive rotation curves (Hindman 1967, Loiseau \& Bajaja 1981). Using a similar technique to derive median radial velocities for HII, Torres \& Carranza (1987) found that the neutral and ionized hydrogen have similar gradients over the main body of the SMC with no important 
systematic differences The question of the meaning of the "rotation curve" is left open because of the many velocity groups not considered in its derivation. Torres \& Carranza also note that a pronounced difference exists between the large-scale motion of the diffuse HII and that of individual HII regions as well as between "field" supergiants and supergiants embedded in HII regions.

Radial velocities of 44 PN in the SMC (Dopita et al. 1985a) show no evidence for organized rotation, nor of any bimodal distribution of the velocities as suggested by Feast (1968) and seen by Torres \& Carranza (1987). If the precursors of the PN are late AGB stars, i.e. carbon (C) stars or luminous MS stars, their distributions should be similar. Hardy et al. (1989) have determined radial velocities for $\mathrm{C}$ stars in three SMC fields. The $\mathrm{C}$ stars do not behave kinematically like the extreme Population I. There is no evidence of a velocity splitting, nor of rotation. The similarity with the PN is evident. The velocity dispersion of the $\mathrm{C}$ stars is the same as that of halo metalpoor giants (Suntzeff $e t$ al. 1986). The C stars and the PN may belong to a spheroidal system which was little affected by the near collision with the LMC $0.4-0.2$ Gyr ago.

\section{The present structure of the Magellanic System}

The global extent of the Magellanic System is enormous (see Mathewson \& Ford 1984, Figs. 12 ). Its total hydrogen mass is estimated to be $1.8 \times 10^{9} \mathrm{M}_{\odot}$ with $5,5,5$, and $1.8 \times 10^{9} \mathrm{M}_{\odot}$ in the $\mathrm{LMC}$, the SMC, the IC region and the Stream, respectively (Kerr 1989). Well over $30 \%$ of the SMC mass and about $5 \%$ of the LMC mass is thus neutral hydrogen.

The structure of the Magellanic System may be analyzed nowadays on the basis of radio, infrared, optical and UV observations. In spite of all the available data (or due to them) the structure of the LMC is a controversial topic and that for the SMC is probably worse.

\subsection{THE MAGELLANIC STREAM AND THE IC REGION}

The Magellanic Stream extends over $100^{\circ}$ from the Clouds along a great circle as seen from the Galactic centre. It is essentially continuous with six main concentrations (Mathewson et al. 1987). No stellar members of the Stream have been found. Interstellar-line observations (Songaila 1981, Penston 1982) show that the Stream cannot be composed of primordial gas. The abundances, slightly below solar, suggest that it originated in the Clouds.

Mathewson et al. (1987) suggested that the IC gas and the Stream were produced in a collision between the LMC and the SMC about 0.4 Gyr ago. The clouds in the Stream formed in collisions between the IC gas and high-velocity clouds in the Galactic halo. A stellar bridge exists between the SMC and LMC (Irwin et al. 1990). Its main-sequence stars have an age of $0.1 \mathrm{Gyr}$, whereas the youngest objects in the Wing are about 5 Myr (Meaburn 1986).

\subsection{THE STRUCTURE OF THE LMC}

The Bar region must have been a major centre of formation of old- and intermediate-age stars. These generations are otherwise found over a much larger area than the youngest one, but their structure, e.g. disk or halo, is not yet well known. Most of the structural peculiarities in the Clouds may be due to close encounters in the past, including the one with our Galaxy when they passed their perigalacticon about 50 Myr ago. The formation of the Extreme Population I started then with almost simultaneous bursts of star formation at several locations. The following SSPSF processes created super-associations with diameters of over $1 \mathrm{kpc}$ (Westerlund 1989). Much of 
their gas and dust has been swept into the delimiting "supergiant shells" (SGSs), (Meaburn 1981). The SGSs contain, apart from young stars and ionized gas, also supernova (SN) remnants and Xray sources (Mathewson et al. 1983). The most typical example is SGS LMC4, connected with Shapley Constellation III (cf. Westerlund \& Mathewson 1966, Meaburn 1981). Its structure has been analyzed by Dopita et al. (1985b). Although its overall structure agrees with the SSPSF model, some modifications appear necessary. Supergiants of ages $1-15$ Myr exist in the field and the majority of the clusters and associations have ages around $4 \mathrm{Myr}$, but the richest stellar arc in the southern part of the SGS is over 20 Myr old. This age distribution may be understood if, during the expansion of the SGS, some clouds were left behind in a weak intercloud medium. In them, star formation was delayed, so that young associations are now found near the site of the origin of expansion and older, more massive ones, in the present SGS.

The youngest region in the LMC, the 30-Doradus nebula, is most likely the result of the collision between two of the shells, SGS LMC 2 and LMC 3 (Westerlund \& Danziger 1985).

If the bursts producing the generations before the LMC had reached a thin-disk structure were followed by SSPSF as in "the Constellation III model" (Dopita et al. 1985b) more stars may have formed away from the plane than in the youngest generation. Once a thin-disk structure had developed, star formation stopped when the gas was thrown out of the plane. This is seen in the velocity dispersions of the supergiants in the superassociations in the LMC. It is small for the KM types, $5.1 \mathrm{~km} \mathrm{~s}^{-1}$ on the average (Prévot et al. 1985). For the OBA stars values over $10 \mathrm{~km} \mathrm{~s}^{-1}$ are derived. This may be due to less accuracy for the early type stars, but it may reflect the fact that they are formed more recently in a more expanded SGS.

The return to the plane of some of the gas, after 0.1 to $2 \mathrm{Gyr}$, has as a main effect an increase of the z- velocity dispersion of the disk HI. It may contribute to the double peaks observed in the $\mathrm{HI}$ velocity curves which is then not completely a sign of a multiple-plane structure (cf. Meaburn $e t$ al. 1987).

The impossibility of having normal spiral arms in the LMC and density waves producing the star formation was shown by Dixon \& Ford (1972). Spiral arms appear, however, often in the discussion of the structure of the LMC; they are sometimes called coherent regular strings (Feitzinger et al. 1987). The spiral structure deduced from the observations of associations, HII regions, UV and IR emission and radio pattern (HI and continua) apears, however, more farfetched than the simple picture of expanding SGSs. At times it also contradicts itself: the existence of "the isolated space-time cell of the Shapley Constellation III" is accepted, but, nevertheless, "the E2 and F strings" include some of the dominating features of this cell. The "octopus" has the head in 30 Doradus but no explanation of its function is found. The structures found in the $1.4 \mathrm{GHz}$ radio continuum pattern can all be fitted to SGSs, preferably by exchanging Meaburn's LMC 3 with one centred at Constellation II, at $05^{\mathrm{h}} 27^{\mathrm{m}}-68.9^{\circ}$, and including most of the associations in the Bar as well as 30 Dor (Westerlund \& Mathewson 1966).

In an interpretation of UV images, Smith et al. (1987) describe the LMC Extreme Population I structure with a deterministic (D) SPSF model. The computed front is made to match spiral arm A (de Vaucouleurs \& Freeman 1973), also arm B is said to match the sequential star-formation process well. However, several of the features that are most prominent in their Figs. 1a and $1 \mathrm{~b}$ (the UV images) are clearly not included in the model and the computed front does not reach 30 Dor.

Thus, the simplest explanation of the present structure of the Extreme Population I in the LMC is the simultaneous bursts of star formation in a number of locations all over the gascontaining area, followed by SSPSF processes. 


\subsection{THE PRESENT STRUCTURE OF THE SMC}

The SMC contains the same age groups as those seen in the LMC. Major differences are: the intermediate-age clusters in the SMC appear older than those in the LMC (Olszewski 1988); the HI distribution is not as clumpy as in the LMC (the clumps surround HII regions and stellar associations); the Bar contains proportionally more blue stars and gas than the LMC Bar, and the Wing exists. Most of the stellar associations of "LMC size" are found in the latter and also the only SGS, SMC1 (Meaburn 1980).

The oldest generation in the SMC may be only 10 to 12 Gyr old (Olszewski 1988) provided RR Lyr stars form later in metal-poor galaxies. Traces of a true Population II have been found (Brück \& Hawkins 1981).

The SMC was first suspected to be appreciably extended in depth by Johnson (1961). Hindman (1967) suggested that, in addition to a flattened system seen edge-one, three expanding shells of gas could be distinguished. Azzopardi (1982) showed that the O, B, A supergiants are spread over $7 \mathrm{kpc}$ in depth with probably two separate groupings.

Recent analysis of the structure of the SMC on the basis of new radio, optical and UV observations show several velocity groups. At least two separate entities of $\mathrm{HI}$ exist, each with its own stellar and nebular populations (Mathewson \& Ford 1984). The idea that the SMC may have been torn apart about 0.2 Gyr ago into a low-velocity fragment (the SMCR), and a higher velocity fragment (the MMC), has been further developed by Mathewson et al. $(1986,1988)$. They found the depth of the SMC to be about $20 \mathrm{kpc}$ with the NE section of the Bar 10-15 kpc closer than the southern. The low-velocity components are closest to us, in contradiction to the conclusions by Caldwell \& Coulson (1986).

In a very extensive investigation, using radial velocities of young stars and HII regions, HI data, and very high spectral resolution profiles of interstellar absorption lines, Martin et al. (1989) find that most of the young stars fall within $10 \mathrm{kpc}$ in depth. The HI in the Bar is distributed in four components, two of which may correspond to the SMCR and MMC. A majority of the stars are associated with one of the four HI components. The main high-velocity complex is located behind the main low-velocity complex. There is high-velocity interstellar calcium gas in front of both these HI complexes. All four components agree with those noted by Torres \& Carranza (1987). A still smaller extension in depth of the SMC has been suggested by Welch et al. (1987), who concluded that the SMC does not extend beyond its tidal radius of the order of $4 \mathrm{kpc}$. Contrary to this, a still larger depth of the SMC has been observed by Hatzidimitriou \& Hawkins (1989).

As a consequence of these contradictory results it may be too early to abandon Hindman's (1967) model with expanding shells with diameters between 1 and $2 \mathrm{kpc}$. Two of the shells (nos. 1 and 3) contain HII regions and young associations, as do the SGSs in the LMC. Hindman's no. 3 shell agrees well with Meaburn's SGS SMC1. If SGSs are accepted as major structural features in the LMC, why should they not exist also in the SMC with a related evolutionary history?

\section{References}

Azzopardi, M. (1982), C.R. Journées Strasbourg 4,20.

Bessell, M.S., Freeman, K.C., Wood, P.R. (1986), Astrophys. J. 310, 710.

Brück, M.T., Hawkins, M.R.S. (1981), IAU Coll. No. 68, A.G.D. Philip, D.S. Hayes (eds) (L.

Davis Press: Schenectady, N.Y.) p.261.

Caldwell, J.A.R., Coulson, I.M. (1986), M.N.R.A.S. 218, 223. 
De Vaucouleurs, G. (1980), Publ. Astron. Soc. Pacific 92, 579.

De Vaucouleurs, G., Freeman, K.C. (1973), Vistas Astron. 14, 163.

Dixon, M.E., Ford, V.L. (1972), Astrophys. J. 173, 35.

Dopita, M.A., Ford, H.C., Lawrence, C.J., Webster, B.L. (1985a), Astrophys. J. 296, 390.

Dopita, M.A., Mathewson, D.S., Ford, V.L. (1985b), Astrophys. J. 297, 599.

Einasto, J., Haud, U., Jôeveer, M., Kaasik, A. (1976), M.N.R.A.S. 177, 357.

Feast, M.W. (1968), M.N.R.A.S. 140, 345.

Feast, M.W., Thackeray, A.D., Wesselink, A.J. (1961), M.N.R.A.S. 122, 433.

Feitzinger, J.V. (1980), Spac. Sci. Rev. 27, 35.

Feitzinger, J.V., Haynes, R.F., Klein, U., Wielebinski, R., Perschke, M. (1987). Vistas Astron. 30, 243.

Feitzinger, J.V., Isserstedt, J., Schmidt-Kaler, Th. (1977), Astron. Astrophys. 57, 265.

Freeman, K.C., Illingworth, G., Oemler Jr., A. (1983), Astrophys. J. 272, 488.

Fujimoto, M., Murai, T. (1984), IAU Symp. 108 Structure and Evolution of the Magellanic Clouds, S. van den Bergh and K.S. de Boer (eds.) (Reidel: Dordrecht) p.115.

Hardy, E., Suntzeff, N.B., Azzopardi, M. (1989), Astrophys. J. 344, 210.

Hartwick, F.D.A., Cowley, A.P. (1988), Astrophys. J. 334, 135.

Hatzidimitriou, D., Hawkins, M.R.S. (1989), Edinburgh Astron. Prep. 9/89.

Hindman, J.V. (1967), Aust. J. Phys. 20, 147.

Irwin, M.J., Demers, S., Kunkel, W.E. (1990), Astron. J. 99,191.

Johnson, H.M. (1961), Publ. Astron. Soc. Pacific 73, 20.

Kerr, F.J. (1989), The World of Galaxies, H.G.Corwin and L. Bottenelli (eds.), p.160.

Kerr, F.J., de Vaucoulers, G. (1956), Aust. J. Phys. 9, 90.

Laney, C.D., Stobie, R.S. (1986), M.N.R.A.S. 222, 449.

Larson, R.B. (1987), Star Bursts and galaxy evolution, T.X. Thuan, T. Montmerle, J.T.T. Van (eds.) p.467.

Lin, D.N.C., Lynden-Bell, D. (1982), M.N.R.A.S. 198, 707.

Loiseau, N., Bajaja, E. (1981), Rev. Mex. Astron. Astrofis. 6, 55.

McGee, R.X. (1964), Aust. J. Phys. 17, 515.

Martin, N., Maurice, E., Lequeux, J. (1989), Astron. Astrophys. 215, 219.

Mathewson, D.S., Ford, V.L. (1984), IAU Symp. 108 Structure and Evolution of the Magellanic Clouds, S. van den Bergh and K.S. de Boer (eds.) (Reidel: Dordrecht) p.125.

Mathewson, D.S., Ford, V.L., Dopita, M.A., Tuohy, I.R., Long, K.S., Helfand, D.J. (1983), Astrophys. J. Suppl. Ser. 51, 345.

Mathewson, D.S., Ford, V.L., Visvanathan, N. (1986), Astrophys. J. 301, 664.

Mathewson, D.S., Ford, V.L., Visvanathan, N. (1988), Astrophys. J. 333, 617.

Mathewson, D.S., Wayte, S.R., Ford, V.L., Ruan, K. (1987), Proc. Astron. Soc. Aust. 7,19.

Meaburn, J. (1980), M.N.R.A.S. 192, 365.

Meaburn, J. (1981), Investigating the Universe, F.D.Kahn (ed.) p.61.

Meaburn, J. (1986), M.N.R.A.S. 223, 317.

Meaburn, J., Marston, A.P., McGee, R.X., Newton, L.M. (1987), M.N.R.A.S. 225, 591.

Meatheringham, S.J., Dopita, M.A., Ford, H.C., Webster, B.L. (1988), Astrophys. J. 327, 651.

Mishra, R. (1985), M.N.R.A.S. 212, 163.

Murai, T., Fujimoto, M. (1980), Publ. Astron. Soc. Japan. 32, 581.

Olszewski, E.W. (1988), The Harlow-Shapley Symp. on Globular Cluster Systems in Galaxies,

J. E. Grindlay and A.G.D. Philip (eds.) p.159.

Page, Th., Carruthers, G.R. (1981), Astrophys. J. 248, 906.

Penston, M.V. (1982), Observatory 102, 174. 
Prévot, L., Andersen, J., Ardeberg, A., Benz, W., Imbert, M., Lindgren, H., Martin, N., Maurice, E., Mayor, M., Nordström, B., Rebeirot, E., Rousseau, J. (1985), Astron. Astrophys. Suppl. Ser. 62, 23.

Prévot L., Rousseau, J., Martin, N. (1989), Astron. Astrophys. 225, 303.

Rohlfs, K., Kreitschmann, J., Siegman, B.C., Feitzinger, J.K. (1984), Astron. Astrophys. 137, 343.

Scalo, J.M. (1987), Star Bursts and galaxy evolution, T.X. Thuan, T. Montmerle, J.T.T. Van (eds.) p.445.

Smith, A.M., Cornett, R.H., Hill, R.S. (1987), Astrophys. J. 320, 609.

Songaila, A. (1981), Astrophys. J. 243, L19.

Songaila, A., Blades, J.C., Hu, E.M., Cowie, L.L. (1986), Astrophys. J. 303, 198.

Suntzeff, N.B., Friel, E., Klemola, A., Kraft, R.P., Graham, J.A. (1986), Astron. J. 91, 275.

Torres, G., Carranza, G.J. (1987), M.N.R.A.S. 226, 513.

Visvanathan, N. (1989), Astrophys. J. 346, 629.

Welch, D.L., McLaren, R.A., Madore, B.F., McAlary, C.W. (1987), Astrophys. J. 321, 162.

Westerlund, B.E. (1989), Recent Developments of Magellanic Cloud Research, K.S. de Boer, F. Spite, G. Stasinska (eds.) p. 159.

Westerlund, B.E. (1990), Astron. Astrophys. Rev. 2, 29.

Westerlund, B.E., Danziger, I.J. (1985), Proc. ESO-IRAM-Onsala Workshop on "(Sub)millimeter Astronomy", P.A.Shaver and K. Kjär (eds.) p.207.

Westerlund, B.E., Mathewson, D.S. (1966), M.N.R.A.S. 131, 371. 